International Journal of Engineering \& Technology, $7(2.8)(2018) 520-523$
International Journal of Engineering \& Technology
SPC
Website: www.sciencepubco.com/index.php/IJET
Research Paper

\title{
Survey on identification and classification of waste for efficient disposal and recycling
}

\author{
AdhithyaPrasanna .M+1*, S. Vikash Kaushal' ${ }^{2}$ P. Mahalakshmi ${ }^{3}$ \\ ${ }^{1,2}$ Student, ${ }^{3}$ Assistant professor OG, SRM Institute of Science and Technology, \\ *Corresponding Author E-mail: adhithyarao96@gmail.com
}

\begin{abstract}
Waste management is a pervasive problem in today's world and is rising continuously with rise in urbanization. For ecologically sustainable development, waste management is a vital requirement in many countries. It is very essential to sort the waste at base level so that there can be proper disposal of waste at the dumping sites. Sorting of waste requires more manpower and consumes more time too. Waste can be sorted and managed in numerous types of techniques. Analysing and classifying the garbage using image processing can be a very productive way to process waste materials. These papers talk about the traditional methods in which waste disposals are taking place. These also talk about the drawbacks faced by the already existing systems and ways to overcome it.
\end{abstract}

Keywords: Waste Classification, Data Mining, Convolution neural Networks, Support Vector Machine

\section{Introduction}

\subsection{Waste Disposal}

Billions of tons of garbage are generated annually across the globe with almost one lakh metric tons of garbage being generated in India per day. Almost $90 \%$ of these wastes are dumped in landfills and water bodies. This can cause a major impact on the environment. The sorting of waste should be done at the earliest stage possible, in order to maximize the amount of recycled items and reduce the possibility of being contaminated by other waste items. Having smarter trash bins helps in this matter by automatically sorting waste on both local and large scales. Recycling of the non-biodegradable waste is currently a manual job.

The present-day process of recycling wastes requires various recycling facilities to handlegarbage and use a combination of large filters and tools to separate out shape specific objects. Improving this process of recycling will help in improving the efficiency of the plant by reducing the wastage, and also in reducing the time consumed in sorting the waste. This will moreover increase the accuracy of the classification compared to that done by hand, and hence will be beneficial in both environmental as well as economical aspects. A stream of images is fed to the system similar to the way it is done in waste management plants. Different objects in the image are identified and each object is classified separately. Image processing algorithm is used to classify the images into six different categories. By automating this process, we can easily classify the recyclable objects by just looking at a single image.

\subsection{Data Mining}

Data mining is the process in which large datasets can be used to efficiently arrive to a proper conclusion about proposed facts supported by precise calculations, and recognize various patterns associated with the domain of datasets used. This can be done by using various methodologies such as statistics, machine learning, pre-existing algorithms defined for specific purposes, and other tools specially designed for this process by experts. It is also possible to provide a valid explanation of facts and their results obtained, and their level of dependency on other facts that affect it. Data mining can be used for processing images along with various tools such as MATLAB, OpenCV and others.

Convolution Neural Network (CNN) are a set of neurons that are interconnected to each other in order to process the input that is being fed into the system and assign logical weights to various parameters that are associated with the data according to their importance and the extent to which they affect the output being produced for the corresponding data and other parameters. It was designed to process data similar to the way the human brain does, and arrive to a conclusion based on the data and the parameters considered. Image processing techniques are highly benefitted by CNN since these can be used on almost all datasets and still can provide accuracy above average for classification and recognition of images. Data mining algorithms such as Support Vector Machine (SVM), k-Nearest Neighbour (kNN), Histogram Equalization and others can also be used for image processing along with the tools preferred for the same. All these algorithms process data fed in different ways and assign weights to parameters based on different criteria, hence exploiting the various possibilities and results.

\section{Existing Systems}

At present different machines[1] are used to separate the waste into different categories. Few of them are-

- Trommel separators/drum screens -Trommel separator contains a rotating drum perforated with holes in it. This separates wastes based on their sizes. When wastes are passed though the 
drum the particles with small size pass out through the holes and large particles stay in the drum.

- Eddy current separator - This system is designed for separation of various metallic materials in wastes. It puts into use an electromagnetic method, dividing the wastes into ferrous and non-ferrous metal categories.

- Induction sorting - This system dispatches the waste materials over a conveyor belt, which has a number of sensors placed under it. The sensor helps in identifying the different types of metals in the wastes. The metals identified are parted by a system consisting of fast air jets which are linked to the corresponding sensors.

- Near Infrared Sensors (NIR) - This system uses the reflectance property as its parameter for distinguishing various waste materials, since different materials exhibit different reflective properties.

- $\quad$ X-ray technology - This system puts into use the density property of various materials for differentiating between them.

- Lastly, the manual method is the most widely used method for separating wastes. Here the wastes are segregated manually by hand.

The above methods can work well in a small scale, but for large scale, these methods are not very effective. It will be difficult to maintain these machines in large scale. Keeping in mind the amount of waste generated now a days, large number of such machines have to be bought. But buying and maintaining these machines will prove to be excessively costly. Also the accuracy of these methods are very low, especially the manual method which is prone to a lot of errors. Making the trash bin smarter provides a better solution to this matter by automatically sorting waste in both home and large scales. Classifying wastes into recycling categories using the technologies available today can prove to be a very efficient methodology to process wastes.

\section{Classification of Images}

\subsection{Classification Based on Shape}

Sergie et al put forward an innovative approach[2] to measure the similarities between various shapes and use the obtained result for recognizing the object. The authors measure the similarity in their framework by solving for concurrence between points chosen on the two shapes considered and by using the conformity to put forth an aligning transform. To solve the symmetry problem, a descriptor is assigned as the shape context to each chosen point. The remaining points are captured relatively to the shape context at the reference point, which offers a unique differentiation characteristic. Corresponding points on two similar shapes will have identical dimensional contexts, hence solving for correspondences. According the point concurrencies, the transfiguration that best coordinates the two shapes is figured out. Transformation maps are provided by regularized thin-plate splines. The deviation between the shapes is estimated as the sum of flaws between the two corresponding points with a terminology that calculates the weightiness of the aligning transform. The identification in a nearest-neighbour classification framework is treated as the problem of deciding the gathered prototype shape that is analogous to that in the image. The result is presented for contours, trademarks, digits, and the COIL dataset. One of the important characteristics of this method is the computation of shape similarity and concurrencies depending on the shape context. This method is comparatively easier to use and simple. It delivers accurate descriptors for point sets that notably improve point set registration, shape recognition and dimensionality matching. This experiment demonstrates variance to several familiar image transformations, including important 3D rotations of real-world objects.

\subsection{Classification Based on Reflectance Properties}

Ron O. Dror et al describe a method [3] that classifies reflectance properties of surfaces under unknown real-world illumination. The surfaces used are metal, plastic and paper. The performance of their algorithm demonstrates the results for surfaces of arbitrary geometry. The reflectance estimation algorithm learns correlations between surface reflectance and the data enumerated from an observed image. It depends on statistical invariability in the spatial structure of real-world lighting. The statistic structure of this algorithm makes it fit better to inaccurate geometries even though known geometry is put into use. This paper puts forward a technique for categorizing using the reflective property of objects, from monochrome images, that is used for surfaces of arbitrary geometry in real-world scenario.

\subsection{Classification Based on Materials}

Ce Liu et al use the Bayesian Framework [4] for classification of the objects based on material recognition. The objects are identified into various material classes like glass, metal, fabric, etc. by processing the surface of a single image respectively. As it is a difficult task to find good, proper, reliable features that can differentiate materials into different categories compared to the traditional methods that are used for object recognition, the researchers used a set of low and mid-level features that are used to learn and obtain various aspects of material appearance. This paper puts forth an augmented Latent Dirichlet Allocation (aLDA) model to combine multiple features under a Bayesian framework to create an ideal combination of features. This system achieved a recognition rate of $44.6 \%$, which is essentially higher than the state of the art which is at $23.8 \%$. The recognition of materials is done by using a Bayesian network and an essential set of realistic parameters. The recognition rate of the proposed model was increased with the help of an aLDA framework. This improved the performance to a greater extent and also enhanced the performance compared to the state of the art model. The contribution of each of the considered features to the performance gain of the system was also analysed by the researchers. This was the first implementation of a model that attempted to recognize the images depending on their materialistic properties.

\subsection{Classification Using CNN}

AlexNet is definitely one of the most popular CNN models used for recognition of images. It was awarded in the 2012 ImageNet Large Scale Visual Recognition Challenge (ILSVRC). AlexNet is widely used owing to its simple and not so deep architecture, along with its efficiency. It is also known to perform well with extremely large datasets. Due to its high efficiency which caused a rise in its popularity, it became a state of the art in classification of images.

Alex Krizhevsky et al trained a large deep convolutional neural network which was used to classify approximately one million high-resolution images into thousand different categories. The test data producederror rates of $37.5 \%$ and $17.0 \%$ on the top- 1 and top-5 respectively,and can be considered as a better approximation compared to the previous models. The neural network had considered 60,000,000 features and consisted of around 650,000 neurons, along with 5 layers of convolution, some of which were supportedby max-pooling layers, and finally 3 fully-connected layers with a1000-way softmax. To reduce overfitting a regularization method called "dropout" was employed. To improve the training speed, non-saturating neurons and a very efficient GPU implementation were used. The results obtained show that a large and deep CNN can bring about very good results on any complicated dataset using supervised learning methods. It is important to be noted that the performance of the whole network deteriorates even if a single convolutional layer is removed. Hence the depth of the whole convolutional neural network plays an important role in achieving good and efficient 
results. The results obtained also showed quite a lot of improvements when the network was trained for a longer time and was made larger, but there is still a long way go in order to match that of the human visual system.

\section{Related Works}

George E Sakr'sresearch[6] aims to automate this process by applying machine learning techniques to recognize the type of waste from their images. Two popular learning algorithms were used: deep learning with convolution neural networks (CNN) and support vector machines (SVM). Each of the algorithms used creates a different classifier that separates waste into 3 main categories: plastic, paper and metal using only $256 \times 256$ coloured png image of the waste. The accuracy produced by the models of the two classifiers were compared in order to choose the best one and the chosen one was implemented on a raspberry pi 3 . The pi controlled a mechanical system that guided the waste from its initial position into the corresponding container of waste to which it was classified. However, George E Sakr et al only compare the two machine learning techniques and implement the best model on the pi in order to measure its speed of classification.

The results obtained show that SVM achieved high classification accuracy of $94.8 \%$ while CNN achieved the same of $83 \%$. SVM also showed an exceptional adaptation to different types of wastes. George E Sakr et al used NVIDIA DIGITS for training the CNN while Matlab 2016a was used to train the SVM. The drawback in George E Sakr's study was the small amount of images in the training set. The training images were scaled down from the original $256 \times 256$ size to $32 \times 32$ size. This reduction contributed to more overfitting problems. The final implemented model produced in this research had very low average execution time (0.1s) on a raspberry pi 3 .

A team created the project AutoTrash [7], an automatic sorting trashcan which seperates trash on basis of compost and recycling properties .The model has a rotating top where a raspberry pi camera is placed. The team used googles tensorflow software and built their own custom layer on top of it for object recognition. The can is partitioned into different areas and the rotating top of the can places the object on their respective areas based on their classification. Auto Trash can only separates the objects into recyclable or compost but classifying into more than two categories would prove to be more useful in the process of recycling.

Spot Garbage [8] is a smartphone based application. It detects a pile of garbage and identifies the location where the garbage is present by using the location access of the smartphones. The app uses the convolutional neural networks architecture for identifying wastes in images. This model estimates an accuracy of $87 \%$ and is trained using GINI (Garbage in images) dataset .This app allows the respective users to report garbage in their nearby places and locations. The authors trained the model by using the patches which were extracted from the Bing Image Searches. This research show cases an architecture o reduce the memory consumed and also reduce the time taken for prediction with zero accuracy loss thus minimizing and optimizing the space consumed in the device used.

Mindy Yang et al researched[9] on classifying garbage into six different classes like metal, paper, cardboard etc. The dataset used was hand collected which consisted of 400 plus images for each of the categories considered. Support Vector Machines with scaleinvariant feature transform (SIFT) features and Convolutional Neural Network were the models that were used to classify the images into various categories.Torch7 framework for Lua was used to construct the $\mathrm{CNN}$ classifier. Eleven layer $\mathrm{CNN}$ architecture was implemented in this, which is very similar to AlexNet. The experiments show that the SVM performs more efficiently than the CNN. $70 \%$ of the data was used to train the model and the remaining $30 \%$ of the data was used to test it. The accuracy obtained was $63 \%$, with the training error resulting in
$30 \%$. Optimal hyper parameters were not obtained and therefore certain categories were omitted to find the optimal accuracy, denoting the fact that the CNN used was not trained to its full potential.

This paper[10] by Maher Arebey et al is about the gray level cooccurance matrix (GLCM) method in image processing domain, which is used in garbage detection and classification. Advanced communication mechanizations were combined with GLCM to enhance the waste assembling operation. Displacement and quantization are the GLAM parameters which were studied to figure out the finest parameter values of the images of wastes. The system proposed in this research utilize various communication technologies such as geographical information system(GIS), radio frequency identification (RFID) and general packet radio system (GPRS) integrated with a normal camera for solving the existing problems and streamlining the solid wastes monitored and efficiently managed. The features are obtained from the GLCM and then used as inputs to the multilayer perceptron (MLP) and Knearest neighbour (KNN) for garbage segregation. The results obtained supports the conclusion made that the KNN classifier out performs the MLP.

Table 1: Garbage Classification models along with their models used and corresponding accuracies

\begin{tabular}{|l|l|l|}
\hline Model & Algorithms used & Accuracy \\
\hline George E Sakr's research & SVM And CNN & $94 \%$ and $83 \%$ \\
\hline Spot Garbage & Convolutional networks & $87 \%$ \\
\hline Mindy Yang et al research & SVM & $63 \%$ \\
\hline
\end{tabular}

\section{Conclusion}

Various steps and methods towards efficient waste management and disposal have already been researched and carried out by many researchers and research enthusiasts. Many devices have also been designed in order to carry on this process efficiently. Hardware components such as raspberry pi have been used along with various algorithms to achieve the goal. Images of objects are scanned using the device in order to classify them accordingly. But a major shortcoming of such devices is that all these devices work with maximum accuracy only on images containing single objects to be recognized and classified. Convolution Neural Networks is considered to play a very important role in the process of object recognition and classification, and can be said to be the major step towards the development of such methods. Methods and steps that consider the shape and size of objects for their recognition and classification have also been developed, but they can be used only on objects that can be said to stay in a specific shape or size, which seems to be a bit difficult when it comes to wastes and scraps. Physical reflectance properties of various objects and also classification of objects based on their materials have also been used earlier to propose methods to classify images accordingly. Previous researches have been more about single object recognition and classification, and moreover, different algorithms were implemented on the same datasets in order to calculate and evaluate the precision and accuracy of the algorithms used to demonstrate the importance and application of the specified algorithm with respect to the kind of datasets used. The major drawbacks of these already existing systems are that they aim to classify only single object in an image. In a real world scenario, it is very difficult to separate individual objects from a garbage pile and then classify them as the amount of garbage present will be in the magnitude of millions of tons and it will be very time consuming. Hence there is a need to recognize and segregate multiple objects in a single image. Also in the current models the number of categories in which wastes are classified are very less. Wastes are usually classified as recyclable and compost. Recyclable materials consists of paper plastic metal etc. and each of it are recycled separately in a different way. Hence to classify the recyclable materials from garbage and wastes into different categories would make the recycling process better and easier. 
From the past decade, there is a huge increase in the use of electronic gadgets, which can be attributed to the explosive growth in computer and technology. The average life of an electronic gadget like smartphone is around 2 years. In the next few decades the amount of electronic wastes generated will increase drastically. It will be very difficult to recycle these components as each part are made of made of different materials and each of these materials have to be recycled in different ways. Therefore an efficient method to recycle electronic gadgets and their spare parts is needed.

\section{Acknowledgement}

We would first like to thank our research advisor Mrs. P. Mahalakshmi of the Computer Science and Engineering Department at SRM University. The door to Asst. Prof. P. Mahalakshmi's office was always open whenever we ran into a trouble spot or had any question about our research or writing. She consistently allowed this survey paper to be our own work, but steered us in the right the direction whenever we needed it.

\section{References}

[1] C. Capel, "Waste sorting - a look at the separation and sorting techniques in todayseuropean market," Waste Management World, 2008

[2] S. Belongie, J. Malik, and J. Puzicha. Shape matching and object recognition using shape contexts. TPAMI, 24(4):509-522, 2002. IEEE TRANSACTIONS ON PATTERN ANALYSIS AND MACHINE INTELLIGENCE, VOL. 24, NO. 24, APRIL 2002

[3] R. Dror, E. H. Adelson, and A. S. Willsky.Recognition of surface reflectance properties from a single image under unknown realworld illumination.In IEEE Workshop on identifyingobjects across variation in lighting, 2001.

[4] C. Liu, L. Sharan, E. H. Adelson, and R. Rosenholtz, "Exploring features in a bayesian framework for material recognition," in Computer Vision and Pattern Recognition (CVPR), 2010 IEEE Conference on. IEEE, 2010, pp. 239-246.

[5] A.Krizhevsky, I. Sutskever, and G. E. Hinton, "Imagene classification with deep convolutional neural networks," in Advances in Neural Information Processing Systems 25,F. Pereira, C. J. C. Burges, L. Bottou, and K. Q.Weinberger, Eds. Curran Associates, Inc., 2012, pp. 1097-1105.

[6] [Online]. Available: http://papers.nips.cc/paper/4824imagenetclassification-

[7] with-deep-convolutional-neural-networks.pdf

[8] Comparing Deep Learning And Support Vector Machines for Autonomous Waste Sorting by George E. Sakr, Maria Mokbel, Ahmad Darwich, Mia Nasr Khneisser, Ali Hadi - 2016 IEEE International Multidisciplinary Conference on Engineering Technology

[9] J. Donovan, "Auto-trash sorts garbage automatically at the techcrunch disrupt hackathon." [Online].Available: https://techcrunch.com/2016/09/13/auto-trash-sortsgarbageautomatically-at-the-techcrunch-disrupt-hackathon/

[10] G. Mittal, K. B. Yagnik, M. Garg, and N. C. Krishnan, "Spotgarbage: Smartphone app to detect garbage using deep learning," in Proceedings of the 2016 ACM International Joint Conference on Pervasive and Ubiquitous Computing, ser. UbiComp '16. New York,NY, USA: ACM, 2016, pp. 940-945. [Online]. http://doi.acm.org/10.1145/2971648.2971731

[11] Mindy Yang , Gary Thung "Classification of Trash for Recyclability Status"Stanford university Available: http://cs229.stanford.edu/proj2016/poster/ThungYangClassificationOfTrashForRecyclabilityStatus-poster.pdf

[12] M A Hannan; Maher Arebey; R. A. Begum; H. BasriGray Leve Aura Matrix: An image processing approach for waste bin level detection, IEEE 2011

[13] T. Padmapriya, V.Saminadan, "Performance Improvement in long term Evolution-advanced network using multiple imput multiple output technique", Journal of Advanced Research in Dynamical and Control Systems, Vol. 9, Sp-6, pp: 990-1010, 2017

[14] S.V.Manikanthan and K.Baskaran "Low Cost VLSI Design Implementation of Sorting Network for ACSFD in Wireless Sensor Network", CiiT International Journal of Programmable
Device Circuits and Systems,Print: ISSN 0974 - 973X \& Online: ISSN 0974 - 9624, Issue : November 2011, PDCS112011008. 